\title{
Falla hepática secundaria a toxicidad por tuberculostáticos. Caso clínico
}

\author{
Fulminant hepatic failure due to tuberculostatic drugs. Case report
}

\author{
Dra. Ivone Malla ${ }^{a}$ Dr. Martín Fauda ${ }^{b}$, Dr. Enrique Casanuevac Dra. María Isabel Fernández ${ }^{d}$, \\ Dr. Marcelo Amante e, Dra. Yu Cheang ${ }^{a}$, Dra. Gisela Giacove ${ }^{a}$, Dra. Alejandra Pedreira ${ }^{a}$, Dr. Pablo Petraccaf, \\ Dr. Ariel González Campaña ${ }^{b}$, Dr. Marcelo Silvag, Dr. Gustavo Podestáb
}

\begin{abstract}
RESUMEN
La toxicidad hepática por isoniacida, sobre todo asociada a rifampicina, es un raro efecto adverso de la terapia antituberculosa. En EE.UU., es la causa de 0,2\% de los trasplantes hepáticos pediátricos y del $14 \%$ de los trasplantes por toxicidad medicamentosa. Comunicamos el caso de una paciente de 10 años de edad con falla hepática fulminante que requirió trasplante hepático luego de cuarenta días de tratamiento tuberculostático con isoniacida, rifampicina y pirazinamida.

Palabras clave: agentes tuberculostáticos, isoniacida, falla hepática aguda, trasplante hepático.
\end{abstract}

\section{SUMMARY}

Hepatoxicity of isoniazid, mainly in association with rifampin, is a rare secondary effect of tuberculostatic treatment. In the United States, it accounts for $0.2 \%$ of all pediatric orthotropic liver transplant, and $14 \%$ of transplants for drug hepatotoxicity. We report the case of a 10 year-old patient who presented with acute liver failure requiring orthotropic liver transplant after forty days of tuberculostatic treatment with isoniazid, rifampin and pyrazinamide.

Key words: antitubercular agents, isoniazid, acute liver failure, liver transplantation.

http:/ /dx.doi.org/10.5546/aap.2012.e35

\section{INTRODUCCIÓN}

La mayoría de los medicamentos puede causar hepatoxicidad. Los más frecuentemente comunicados son los antiinflamatorios no esteroideos, antibióticos, antiepilépticos, estatinas, tuberculostáticos y hierbas medicinales. ${ }^{1}$

La definición de falla hepática aguda, originalmente descripta en adultos, ${ }^{2}$ incluía como requisito para su diagnóstico la presencia de encefalopatía. En niños, es definida por Bhaduri y Mieli-Vergani como un trastorno multisistémico con grave afectación de la función hepática, con encefalopatía o sin ella, en un paciente sin enfermedad hepática crónica conocida. ${ }^{3}$ El trasplante hepático es la herramienta terapéutica que ha logrado mejorar su alta mortalidad.

Para determinar qué pacientes requerirán trasplante hepático se utilizan los criterios pronósticos del Kings College, ${ }^{4}$ definidos para toxicidad por paracetamol: 1) pH menor a 7,30,2) relación internacional normatizada (RIN) mayor a $6,5,3$ ) creatinina mayor a $3 \mathrm{mg} / \mathrm{dl}$ o 4) encefalopatía de grado III-IV; y para otras etiologías: RIN mayor a 6,5 como criterio único o tres de: 1 ) edad menor de 10 o mayor de 40 años, 2) etiología hepatitis noA-E, fármacos, 3) duración de la ictericia previo a la encefalopatía mayor a 7 días, 4) RIN mayor a 3,5 o (5) bilirrubina sérica mayor a $17,5 \mathrm{mg} / \mathrm{dl}$.

En nuestro país, las causas de trasplante hepático por falla hepática fulminante cambiaron desde la introducción de la vacuna antihepatitis A en el calendario oficial. En el período previo a esta incorporación, la hepatitis fulminante por virus A representaba el 30\% de los trasplantes hepáticos pediátricos. ${ }^{5}$ Desde el comienzo de la vacunación universal en 2005 hubo una caída brusca de casos de hepatitis A y, desde 2007, no hay informes de trasplante hepático pediátrico por esta causa. Acompañando a este cambio epidemiológico, las causas de trasplante hepático por falla hepática fulminante se modificaron y adquirieron relevancia las autoinmunitarias, criptogénicas y tóxicas. 
Altos niveles de transaminasas e ictericia inducidas por fármacos se asociaron con alta mortalidad en observaciones de Zimmerman en la década de 1970 y en informes posteriores. ${ }^{6,7}$

La isoniacida es un antibiótico sintético introducido en 1952 que actúa inhibiendo pasos oxígeno-dependientes en la síntesis del ácido micólico, un componente de la pared celular micobacteriana. Causa dos reacciones adversas mayores: neurológica, por competencia con piridoxina (vitamina $\left.\mathrm{B}_{6}\right),{ }^{8}$ y hepática. Otros efectos comunicados con menor frecuencia son el síndrome de tipo lupus y la hipersensibilidad al fármaco. La toxicidad neurológica se manifesta por neuropatía, habitualmente con entumecimiento, hormigueo o dolor urente en extremidades, rara vez con ataxia y nistagmus. ${ }^{9}$ El mecanismo del daño hepático no está completamente claro, la apariencia histopatológica es similar a la de las hepatitis virales, no se relaciona con las concentraciones séricas del fármaco o con autoanticuerpos, y no hay evidencia de que reacciones alérgicas o de hipersensibilidad jueguen algún rol. La isoniacida es acetilada e hidrolizada en el hígado, con producción de metabolitos implicados en la patogenia de la hepatotoxicidad. También puede oxidarse vía el citocromo $\mathrm{P} 450$ para formar radicales reactivos. La rifampicina incrementa la actividad del citocromo P450 y, por lo tanto, puede aumentar la producción de los metabolitos tóxicos de la isoniacida. ${ }^{10}$ En caso de lesión hepática grave existen diferentes informes sobre trasplante hepático, llevado a cabo aun con tuberculosis pulmonar activa. ${ }^{11,12}$

En EE.UU., la intoxicación por paracetamol es la principal causa de hepatitis fulminante..$^{13} \mathrm{La}$ toxicidad por isoniacida es la causa de $0,2 \%$ de los trasplantes hepáticos pediátricos y del $14 \%$ por toxicidad medicamentosa. ${ }^{11}$

Comunicamos el caso de una paciente de 10 años de edad quien sufrió falla hepática fulminante luego de cuarenta días de tratamiento con isoniacida, rifampicina y pirazinamida y requirió trasplante hepático.

\section{CASO CLÍNICO}

Se presentó en nuestro centro una niña de 10 años de edad, que había estado en contacto con un portador de tuberculosis pulmonar, bacilífero (su hermano de 15 años), a quien se le realizó radiografía de tórax que fue normal y prueba cutánea de derivado proteico purificado (PPD) con pápula mayor a $15 \mathrm{~mm}$, por lo cual comenzó tratamiento con rifampicina (10 mg/ $\mathrm{kg} /$ día), isoniacida ( $5 \mathrm{mg} / \mathrm{kg} /$ día) y pirazinamida (30 mg/ $\mathrm{kg}$ / día). Cuarenta días luego del comienzo del tratamiento presentó vómitos gástricos, a los que se agregó fiebre, astenia, hiporexia, ictericia, disartria y un episodio de convulsión tónico-clónica generalizada de una hora y media de duración. Fue medicada con benzodiacepinas y difenilhidantoína. Al ceder el episodio convulsivo, se realizó tomografía de cerebro y punción lumbar, ambas con resultados normales y cultivo de líquido cefalorraquídeo negativo. Se constató ictericia, alteración de aspartato aminotransferasa (AST) y alanino aminotransferasa (ALT), anteriormente llamadas transaminasa glutámico oxalacética (GOT) y transaminasa glutámico pirúvica (GPT), respectivamente. La paciente tenía AST de 28 y ALT de 37 veces el valor normal, bilirrubina 13 veces el valor normal y coagulopatía (RIN 8,51 valor de referencia 1-1,3), con encefalopatía que requirió ventilación mecánica. Se suspendió la medicación tuberculostática y se implementaron medidas de sostén incluyéndose en lista de trasplante hepático.

Ante la neurotoxicidad probablemente asociada a isoniacida, manifestada con estatus epiléptico, se indicó vitamina $\mathrm{B}_{6}$. Con electroencefalograma normal, se suspendió la medicación anticonvulsivante.

Con el objetivo de descartar tuberculosis activa, se realizó tomografía de cerebro, tórax y abdomen, constatándose como único hallazgo una imagen quística, lobulada, en riñón izquierdo. Se tomó muestra de orina, que no mostró piuria, con $\mathrm{pH}$ de 7. Se realizó punción aspirativa, con rescate de líquido no caseoso y cultivo para micobacterias negativo, interpretándose como quiste renal simple.

Se estudiaron otras causas de lesión hepática y se descartaron hepatitis virales, autoinmunitarias, enfermedad de Wilson, déficit de alfa1 antitripsina, hemocromatosis, celiaquía, metabolopatías, enfermedades bacterianas y parasitarias.

En evaluación por el Servicio Social se detectó un grupo familiar numeroso, con necesidades básicas insatisfechas, vivienda precaria que no cubría las necesidades ambientales para el postrasplante, con un padre que ejercía violencia física, emocional y económica. Vínculos familiares quebrados, sin red social de apoyo y contención. La madre registraba la gravedad del cuadro de su hija y podía tomar decisiones. Ambos progenitores inmigrantes, el padre albañil, con problemas laborales por etilismo y la madre empleada doméstica.

A las $48 \mathrm{~h}$ del ingreso, recibió trasplante hepático ortotópico con donante cadavérico. La 
evaluación histopatológica del hígado explantado mostró necrosis hepática masiva con compromiso mayor al $95 \%$ de la superficie de corte (Figura 1). Se utilizó inmunosupresión con corticoides y tacrólimus a dosis habituales. El período posquirúrgico no presentó complicaciones y fue externada a los 10 días postrasplante. Se decidió continuar terapia tuberculostática con ciprofloxacina y etambutol hasta completar seis meses de tratamiento. Se trabajó con el Servicio Social en mejoras de la vivienda y condiciones seguras para su seguimiento.

\section{DISCUSIÓN}

En la Argentina, la tasa para todas las formas de tuberculosis en 2009 fue de 26,6 casos por 100000 habitantes, $10 \%$ de los cuales se presentaron en menores de 14 años. ${ }^{14}$

Ante la prevalencia de tuberculosis en nuestro país, resulta de suma importancia considerar los efectos tóxicos de la medicación, entre ellos la hepatotoxicidad. La insuficiencia hepática por toxicidad debido a isoniacida en el tratamiento tuberculostático es un cuadro raro con elevada mortalidad. ${ }^{11}$

Todo paciente con alteración grave de la función hepática, sin enfermedad hepática crónica conocida y con coagulopatía (tiempo de Quick inferior a $60 \%$ ) que no mejora con vitamina $\mathrm{K}$, debería ser trasladado a un centro de mayor complejidad para evaluar la necesidad de su trasplante hepático.
En el caso presentado, siguiendo pautas de diagnóstico de hepatotoxicidad por fármacos ${ }^{15}$ y criterios de pronóstico de falla hepática fulminante, ${ }^{4}$ luego de descartar otras etiologías, con parámetros serológicos de riesgo de mortalidad, ${ }^{7,8}$ indicamos trasplante hepático.

El medio social era conflictivo y la única figura de contención era la madre de la paciente, motivo por el cual no fue posible plantear la alternativa de donante vivo. Esta circunstancia generó un fuerte trabajo para mejorar la vivienda y las condiciones de cuidado en el postrasplante.

Por otro lado, el antecedente de contacto con tuberculosis y una PPD positiva, hicieron necesaria la búsqueda exhaustiva de enfermedad activa y la continuación del tratamiento con fármacos alternativos, por el riesgo de desencadenar infección al momento de indicar inmunosupresión para el trasplante.

Creemos que este informe es de utilidad para mantener presente la posibilidad de lesión hepática grave causada por fármacos, además de plantear las dificultades sociales que habitualmente se manifiestan al momento de decidir un trasplante hepático.

\section{BIBLIOGRAFÍA}

1. Lee WM. Drug-induced hepatotoxicity. $N$ Engl J Med 1995;333(17):1118-27.

2. Bernau J, Rueff B, Benhamon JP. Fulminant and Subfulminant Liver Failure: Definitions and Causes. Semin Liver Dis 1986;6(2):97-106.
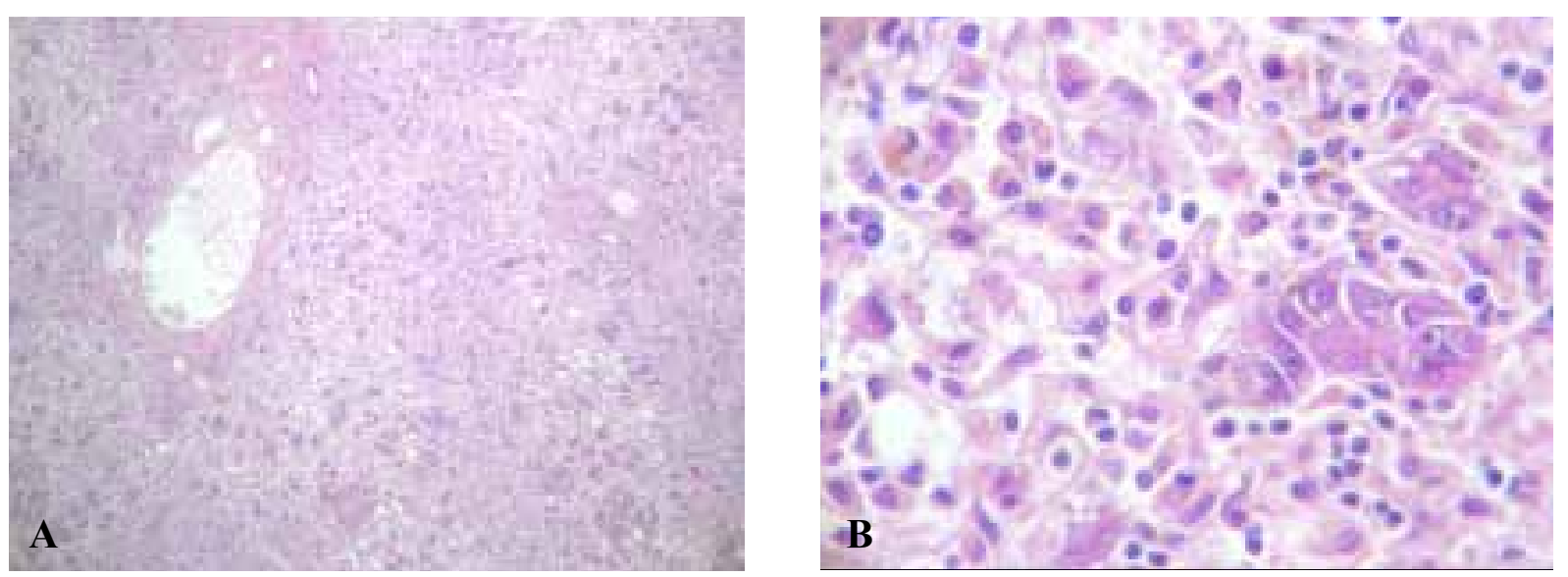

A. Extensas áreas de necrosis confluente (hematoxilina-eosina, 40x).

B. Infiltrado inflamatorio en espacios porta (hematoxilina-eosina, 100x). 
3. Bhaduri and Mieli-Vergani. Fulminant hepatic failure: pediatric aspects. Semin Liver Dis 1996;16(4):349-55.

4. O'Grady JG, Alexander GJ, Hayllar KM, Williams R. Early indicators of prognosis in fulminant hepatic failure. Gastroenterol 1989;97:439-45.

5. Ciocca M. Clinical course and consequences of hepatitis A infection. Vaccine 2000;18(Suppl 1): S71-4.

6. Zimmerman HJ. Various forms of chemically induced liver injury an their detection by diagnostic procedures. Environ Health Perspect 1976;15:3-12.

7. Li B, Wang Z, Fang J, Xu C, et al. Evaluation of prognostic markers in severe drug-induced liver disease. World J Gastroenterol 2007;13(4):628-32.

8. Prevention and treatment of tuberculosis among patients infected with human immunodeficiency virus: Principles of therapy and revised recommendation. Centers for Disease Control and Prevention. MMWR Recomm Rep 1998;47(RR20):1-58.

9. Lewin PK, McGreal D. Isoniazid toxicity with cerebellar ataxia in a child. CMAJ 1993;148(1):49-50.
10. Steele MA, Burk RF, DesPres RM. Toxic hepatitis with isoniazid and rifampin. A meta-analysis. Chest 1991;99(2): 465-71.

11. Wu SS, Chao CS, Vargas JH, et al. Isoniazid related hepatic failure in children: a survey of liver transplantation centers. Transplantation 2007;84(2):173-9.

12. Farrel FJ, Keeffe EB, Man KM, Imperial JC, et al. Treatment of hepatic failure secondary to isoniazid hepatitis with liver transplantation. Dig Dis Sci 1994;39(10):2255-9.

13. Koffron A, Stein JA. Liver Transplantation: indications, pretransplant evaluation, surgery, and posttransplant complications. Med Clin N Am 2008;92(4):861-88.

14. Ministerio de Salud, Presidencia de la Nación Argentina. Notificación de casos de Tuberculosis en la República Argentina. 1980-2009. Buenos Aires, 2010. [Acceso: 16 jun 2011]. Disponible en: http://www.anlis.gov.ar/inst/iner/ archivos/NotificacionTB_09.pdf]

15. Teschke R. Hepatotoxicity by drugs and dietary supplements: safety perspectivas on clinical and regulatory issues. Ann Hepatol 2009;8(3):184-95. 